MICRON-SCALE DEEP HOLE

DRILLING FOR BERYLLIUM

CAPSULE FILL APPLICATIONS

J. P. Armstrong, A. M. Rubenchik, J. Gunther, B.

C. Stuart

January 6, 2006

Fusion Science And Technology 
This document was prepared as an account of work sponsored by an agency of the United States Government. Neither the United States Government nor the University of California nor any of their employees, makes any warranty, express or implied, or assumes any legal liability or responsibility for the accuracy, completeness, or usefulness of any information, apparatus, product, or process disclosed, or represents that its use would not infringe privately owned rights. Reference herein to any specific commercial product, process, or service by trade name, trademark, manufacturer, or otherwise, does not necessarily constitute or imply its endorsement, recommendation, or favoring by the United States Government or the University of California. The views and opinions of authors expressed herein do not necessarily state or reflect those of the United States Government or the University of California, and shall not be used for advertising or product endorsement purposes. 


\title{
MICRON-SCALE DEEP HOLE DRILLING FOR BERYLLIUM CAPSULE FILL APPLICATIONS
}

\author{
J.P. Armstrong, A.M. Rubenchik, J. Gunther, and B.C. Stuart \\ Lawrence Livermore National Laboratory \\ PO Box 808, Mail Stop L-479, Livermore, CA 94550 \\ Email: armstrong16@1lnl.gov
}

\begin{abstract}
A laser processing system has been developed to drill high aspect ratio holes through the impermeable beryllium capsules envisioned for ignition shots on NIF. The drilling system was designed to produce holes with an entrance and exit diameter of approximately $5 \mu \mathrm{m}$ through the full $175 \mu \mathrm{m}$ thickness of the capsule. To meet these requirements, a frequency doubled femtosecondclass Ti:Sapphire laser is directed through a high numerical aperture lens to provide the spot geometry needed to drill the hole. The laser pulse is confined by the metallic walls of the hole, thereby maintaining the diameter of the channel well beyond the Rayleigh range of the optical system. Presented is the current state of this work-in-progress, including descriptions of the device and the technique used to produce the holes. The various means of characterizing the laser-drilled channels are also discussed.
\end{abstract}

\section{INTRODUCTION}

One of the leading ignition target designs ${ }^{1}$ for the National Ignition Facility (NIF) ${ }^{2}$ requires a copper-doped beryllium ablator. Unlike plastic or glass, this material is impermeable to hydrogen, requiring that a hole be used to fill the capsule with DT fuel. The requirements for this hole are quite stringent because density perturbations in the ablator can enhance instability growth during an implosion, which in turn reduces the fusion yield. Recent calculations suggest that $5 \mu \mathrm{m}$ diameter hole through the full thickness of the capsule would not require a Be plug to correct the mass defect. ${ }^{3}$

Several technologies have been explored in the past for drilling holes of similar dimensions. These techniques include electrical-discharge machining (EDM) and focused ion beam (FIB) milling. The main issue encountered with these technologies is a limit in aspect ratio (ratio of hole depth to hole diameter) for holes that are less than $10 \mu \mathrm{m}$ in diameter. Generally speaking for common materials, aspect ratios greater than 18 are difficult to attain with holes this small using EDM. ${ }^{4}$
Although aspect ratios for FIB milling as high as 60 have been reported for specific, porous materials, ${ }^{5}$ conventional FIB milling has a practical limit of approximately 12 for solid density metals (such as beryllium) before taper between the entrance and exit dimensions becomes a concern. The proposed fill hole in this application represents an aspect ratio of 35 .

The proposed fill-tube baseline design ignition capsule consists of a beryllium ablator with a gradient doping of copper. The $175 \mu \mathrm{m}$ thick $\mathrm{Be}-\mathrm{Cu}$ ablator is deposited $^{6,7}$ on a $15 \mu \mathrm{m}$ thick $\mathrm{CH}$ mandrel $^{8}$ that is removed through pyrolysis ${ }^{9}$ after the fill hole is drilled. Once drilled and pyrolized, a $12 \mu \mathrm{m}$ diameter counterbore up to $20 \mu \mathrm{m}$ deep will be machined over the fill hole and a $10 \mu \mathrm{m}$ fill tube is then inserted and epoxied in place.

Prior work in femtosecond materials processing has demonstrated the ability to precisely remove material with a non-thermal, ablative mechanism in a large variety of materials. ${ }^{10-14}$ In this regime a majority of the laser pulse is absorbed within an optical skin depth $(\sim 10 \mathrm{~nm}$ for beryllium at $400 \mathrm{~nm}$ ) of the metal being processed. The laser energy is coupled to the electrons on a time scale that is much faster than that of the energy transfer to the bulk of the material. The result is the formation of a plasma with much higher initial energy than would be found in an evaporative (thermal) process. This increased energy aids in the removal of the material from the long, narrow channel.

\section{FILL HOLE FORMATION AND LASER CONFINEMENT}

Previous work $^{15,16}$ has shown that deep channel formation is an exceedingly complex process. At the beginning of the drilling process, the laser pulse strikes the clean surface of the material and forms a hole several nanometers deep. As the depth increases the surface morphology of the hole bottom begins to roughen, leading to a decrease in the amount of material removed per 
pulse. The rough texture leads to the formation of multiple channels (filaments) that are many times smaller than the diameter of the hole. These channels appear to have an increased ablation rate relative to that of the remainder of the hole bottom. The filaments break through the back surface first, leading to complex exit morphology if drilling is ceased immediately.

The extremely small beam diameter needed for micron-scale hole drilling requires a beam that is inherently highly divergent from the focal spot. Figure 1 shows the theoretical beam profile near focus for the final lens used in these experiments, assuming diffraction limited performance of the entire system and a $400 \mathrm{~nm}$ laser with perfect beam quality $\left(\mathrm{M}^{2}=1\right)$.

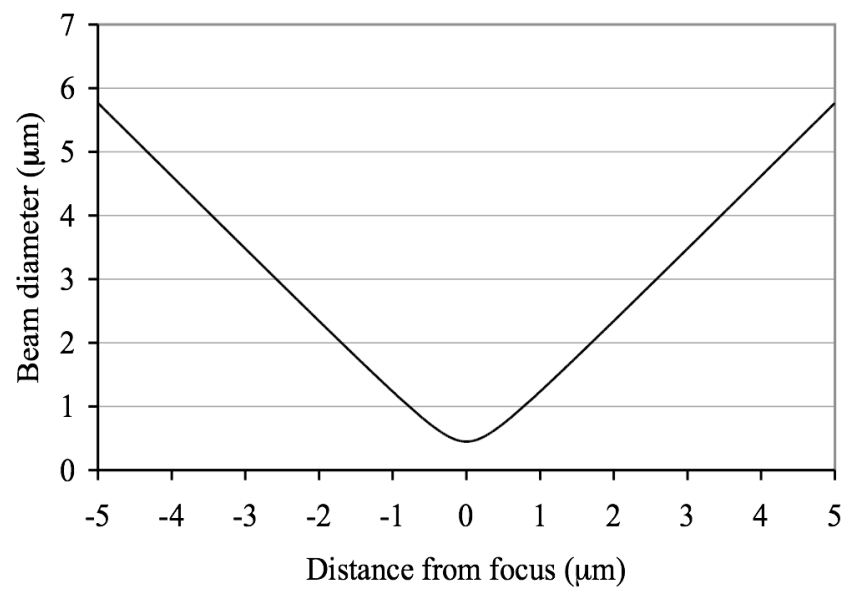

Figure 1. Theoretical free space beam propagation profile near the focus using final lens and $400 \mathrm{~nm}$ laser.

The beam propagation shown in Fig. 1 is useful for understanding the sensitivities and ramifications of using high numerical aperture (NA) lens. First, placing the part precisely at focus is difficult because the depth of focus (defined as the axial distance where the beam radius lies within a factor of $\sqrt{2}$ of its minimum value) for the system is less than one micron. Additionally, note that if the hole diameter followed the profile shown, the maximum depth one could attain before the hole no longer met specifications would be just over four microns. Instead, the drilling process relies on the fact that light incident on a metallic surface at high angle will reflect off that surface. By launching the light into the metallic cylinder (hole), the beam is confined in a waveguide in much the same way as it would be in an optical fiber.

Light propagation in metallic waveguides and the effects of focusing conditions on propagation has been studied previously. ${ }^{[1]}$ Although the experimental conditions between this work and that in Ref. 17 differed in hole diameter, wavelength and pulse duration, the underlying concepts of optical confinement and attenuation are relevant and can be used as a basis for future modeling specific to our circumstances. Figure 2 depicts a basic ray model of confinement and defines the parameters relevant to subsequent discussions.

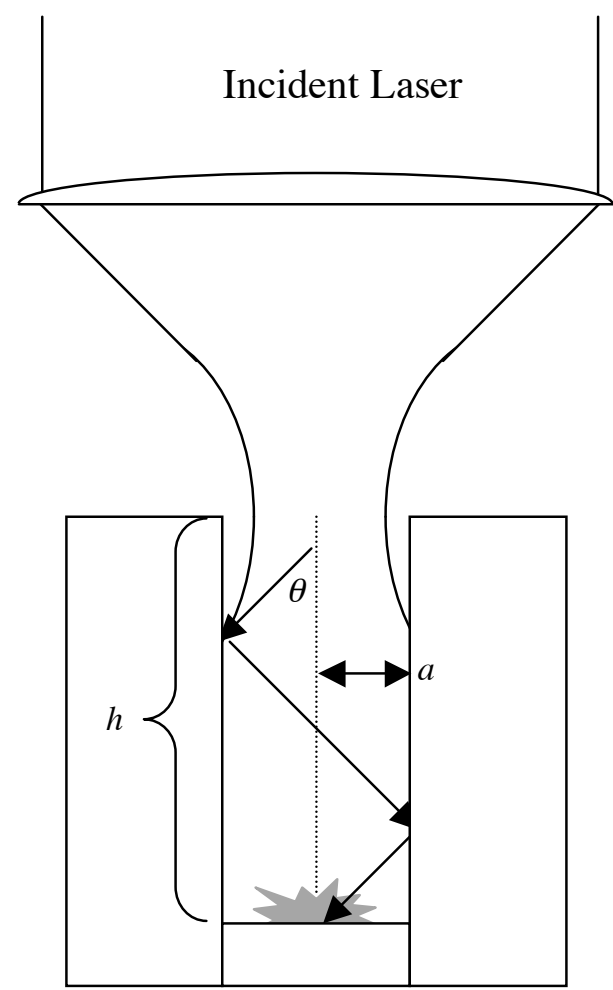

Figure 2. Depiction of the metallic waveguiding ray model parameters.

The relevant parameters shown in Fig. 2 are defined as follows:

$$
\begin{aligned}
& \theta=\text { largest incident angle of light on hole wall } \\
& h=\text { depth of hole } \\
& a=\text { radius of hole }
\end{aligned}
$$

Three equations that are associated with the model that aid in the understanding of the waveguiding tradespace are shown below.

$$
\begin{gathered}
\theta \approx \frac{1}{k \cdot a} \\
N=\frac{\theta \cdot h}{a} \\
\alpha=\frac{\operatorname{Re}(\xi) \cdot \theta}{|\xi|^{2}}
\end{gathered}
$$

where

$k=$ wavenumber of light

$N=$ number of reflections the light goes through to reach hole bottom

$\alpha=$ attenuation of beam on each reflection

$\xi=$ impedance of the material being drilled 
The ray model is sufficient to conceptually describe several phenomenon observed during hole processing. First, each reflection off the hole wall decreases the laser intensity $(\alpha)$. Total attenuation of the beam increases with increasing hole depth and decreasing hole diameter (due to the increasing number of reflections down the channel and the larger incident angle on the hole wall). Drilling stalls for a specific input energy when loss due to multiple reflections $(N \cdot \alpha)$ is sufficient to drop the laser intensity below ablation threshold at the bottom of the hole. The laser intensity may be increased to drill deeper, but the hole enlarges as more of the focal spot profile increases above ablation threshold. This enlargement necessitates using a smaller focused spot size than the desired hole size. The laser attenuation with increasing depth in combination with the ability of the plasma to escape the channel before cooling to the condensation point limits the attainable aspect ratio.

\section{APPARATUS AND PROCESS}

Prior experience suggested that a femtosecond laser might be ideal for micron-scale, high aspect hole drilling because of the large kinetic energy imparted to the small amount of material being removed. The laser source was a generic chirped-pulse amplification ${ }^{18}$ Ti-Sapphire system designed by LLNL. In summary, the system provided a pulse energy of $1 \mathrm{~mJ}$ in a $130 \mathrm{fsec}$ pulse duration at a repetition rate of $3.5 \mathrm{kHz}$. This compressed pulse was frequency doubled to provide $45 \mathrm{~mJ}$ of $400 \mathrm{~nm}$ light. The beam was collimated to a $1 / \mathrm{e}^{2}$ diameter of 4.6 $\mathrm{mm}$ and passed through a zero-order $\lambda / 2$ waveplate and Glan-laser polarizer to provide a continuously variable pulse energy. Finally, a $\lambda / 4$ waveplate was used to place the laser in a circular state of polarization to avoid static linear polarization effects. ${ }^{19}$

The design of the drilling station itself began with choosing the beam size that would be required on the part. Early experiments showed that a spot size of less than one micron would be required to allow sufficient room for growth of the hole as the intensity is increased to overcome waveguide losses. The lens chosen for the apparatus was an aspheric single-element lens with a focal length of $4 \mathrm{~mm}$ and a clear aperture of $4.6 \mathrm{~mm}(\mathrm{NA}=$ 0.58). This objective, in combination with the $400 \mathrm{~nm}$ light sized to the full clear aperture of the lens would produce a spot diameter of $450 \mathrm{~nm}$ with a depth of focus of $770 \mathrm{~nm}$ in the diffraction limit. The actual spot diameter at the focus is enlarged due to aberrations in the optical system and imperfections in the laser. SEM images show that the system is capable of producing a hole on the surface of a properly positioned target that is approximately $0.9 \mu \mathrm{m}$ in diameter when exposed with $100 \mathrm{~nJ}$ of energy.
The combination of the lens and short pulse duration of the laser had several impacts on the design of the rest of the system. First, drilling operations had to be done in vacuum since the system was able to create intensities on the order of $10^{14} \mathrm{~W} / \mathrm{cm}^{2}$ at the focal spot. Beam intensities this high lead to air breakdown and can cause nonlinear phase aberrations. The short working distance of the objective $(2.4 \mathrm{~mm})$ necessitated the lens being housed inside the vacuum system as well. Secondly, the proximity of the lens to the cutting site and the high degree of directionality of the plasma exiting the channel would mean that significant debris would be aimed toward the lens. This particular lens was designed to compensate for $250 \mu \mathrm{m}$ of glass $(n=1.523)$ between the lens and the focal spot, enabling the insertion of a microscope cover slip to act as a sacrificial debris shield. Lastly, the short depth of focus would require very precise positioning of the part along the propagation axis (z-axis).

Positioning the hole on the target in three dimensions while allowing for variations in the capsule diameter, capsule wall thickness and deformation of chamber components during pump down required the use of a sensor that utilized the drilling optical system and capsule for feedback. To accomplish this, a second laser was aligned down the optical axis of the final lens to act as an illumination source. The illumination source's wavelength $(635 \mathrm{~nm})$ was chosen such that dichroic mirrors could easily separate the cutting wavelength and the illumination wavelength. The illumination source propagated through the final lens and came to a focus on the capsule. The light was reflected by the specular surface of the capsule and was recollimated by the objective. By placing a second lens (tube lens) beyond the injection point of the laser and monitoring the diameter of the focal spot with a CCD camera, we created a confocal sensor that produced a spot of minimum diameter only when the part was precisely at focus. Figure 3 shows a simple schematic of the Confocal Placement Sensor (CPS) and the location of the cutting laser injection point.

Proper functionality of the focal sensing system depended on several factors. First, the target being cut must be a specular reflector. Fortunately, the capsules being drilled are required to have excellent surface quality to ensure high fusion yield. Secondly, the relative divergence between the illumination laser and the cutting laser must always remain constant. This can be difficult to accomplish because the divergence of each laser source can be affected in varying degrees by several dynamic factors, including ambient temperature fluctuations, laser warmup time, and gradual aging of the lasers. To overcome these issues, the illumination divergence was forced to be constant by fiber delivering it to the system. In addition, the divergence of the cutting laser was 


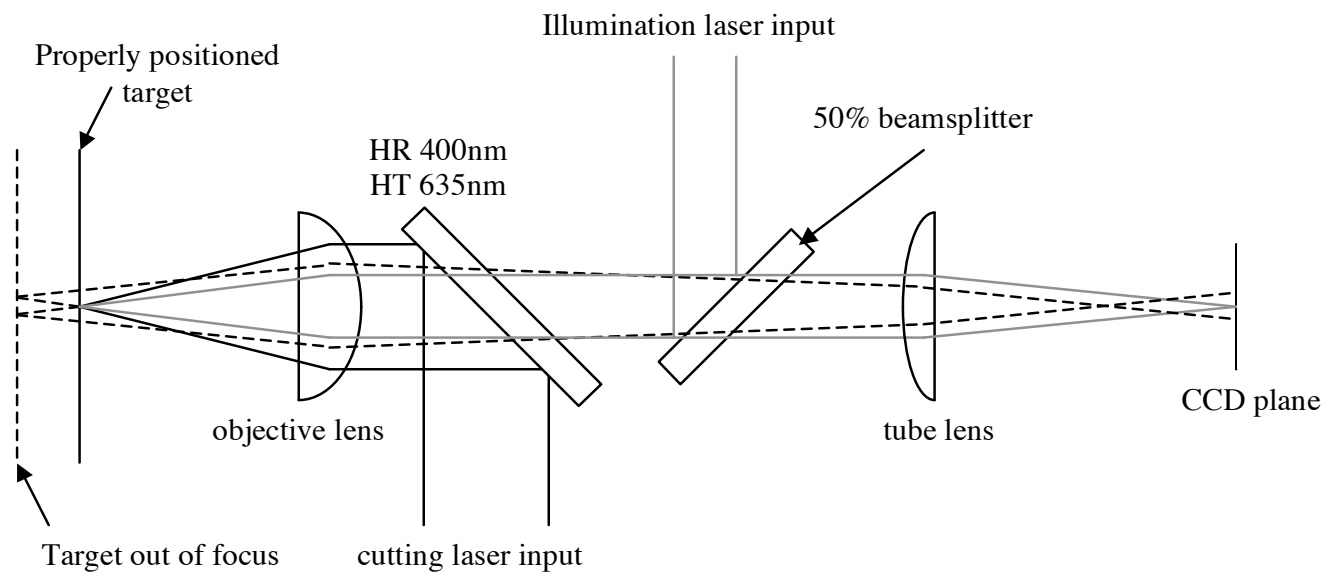

Figure 3. Diagram showing the basic optical system for cutting-laser injection and focal sensing. A target positioned on either side of focus results in an enlarged spot on the CCD camera. The gray line indicates ray path for properly positioned target while the dashed line shows ray path for a target that is out of focus.

checked and adjusted prior to each drilling session using a Hartmann wavefront analyzer. The final caveat to optimal system performance is to hold the relative mechanical motion between each optical component to an absolute minimum. This apparatus utilized a continuous cage structure that extends on both sides of the vacuum barrier to hold each component. Measurements made on the accuracy of the CPS with ideal surface quality demonstrated $z$-axis positioning of the part within $1 \mu \mathrm{m}$ of best focus.

Our CPS allowed the use of an additional diagnostic that has proven to be extremely valuable. A high resolution video microscope was created by intentionally adding extra divergence in the illumination source to flood a larger spot on the sample and shifting the camera to the image plane. This configuration provided a fieldof-view (FOV) of $75 \mu \mathrm{m}$ with a resolution of approximately $300 \mathrm{~nm} /$ pixel. The added divergence on the illumination beam shifted the apparent focal point on the CPS, which was corrected by translating the CCD to the new location. Adding a beamsplitter and a second camera after the tube lens allowed simultaneous monitoring of the image plane (microscope providing $x$ and $y$-axis part positioning) and the focal plane (confocal sensor providing $z$-axis positioning).

The part-positioning specifications required coupling a high-performance translation system with motorized replacement actuators (MRA) that had very small minimum incremental motion. All of the motion equipment had to be rated for vacuum at levels less than 10 mTorr. The MRAs (Picomotor MRA, New Focus) provided a minimum incremental motion of about $30 \mathrm{~nm}$ when pushing the maximum specified load of $22 \mathrm{~N}$. The relatively small load capacity required a custom set of vacuum compatible stages with low spring-rate preloads and the complete removal of the $z$-axis (axis alligned to gravity) spring. The monolithic 3 -axis stage was a stainless steel, crossed-roller bearing design typically used for fiber optic alignment (561D-XYZV, Newport Corporation). Figure 4 shows a sectioned view of the asbuilt apparatus.

The drilling process was developed over several years of experimentation. These experiments yielded a set of laser parameters and exposure times that are known collectively as the "standard drill tool." This parameter set was used for $175 \mu \mathrm{m}$ thick beryllium to produce a minimum diameter hole while still ensuring breakthrough of the capsule wall. This parameter set is used when no method of breakthrough detection is available, as was the case for a spherical capsule. Although the system is capable of producing smaller holes, this standard drill tool increases the energy beyond the breakthrough threshold so that the process is relatively insensitive to many variables including fluctuating laser energy, absorption due to debris shield contamination and placement of the part slightly out of the focal plane of the lens. Experience has also shown the debris shield should be moved or changed after drilling no more than five holes of $175 \mu \mathrm{m}$ thick beryllium to avoid having contamination affect hole quality and depth.

The standard drill tool begins with positioning the part in the $x$-axis and $y$-axis such that the drilling crosshair is positioned at the proper location the part. Focus is then verified using the CPS by translating the part along the propagation axis ( $z$-axis) and minimizing the focal spot diameter on the CPS camera. Drilling begins with an initial exposure while the energy control is set to its minimum $(100 \mathrm{~nJ})$. Proper part positioning is immediately verified by the appearance of a very small diameter hole (less than $1 \mu \mathrm{m}$ ) in the microscope camera 


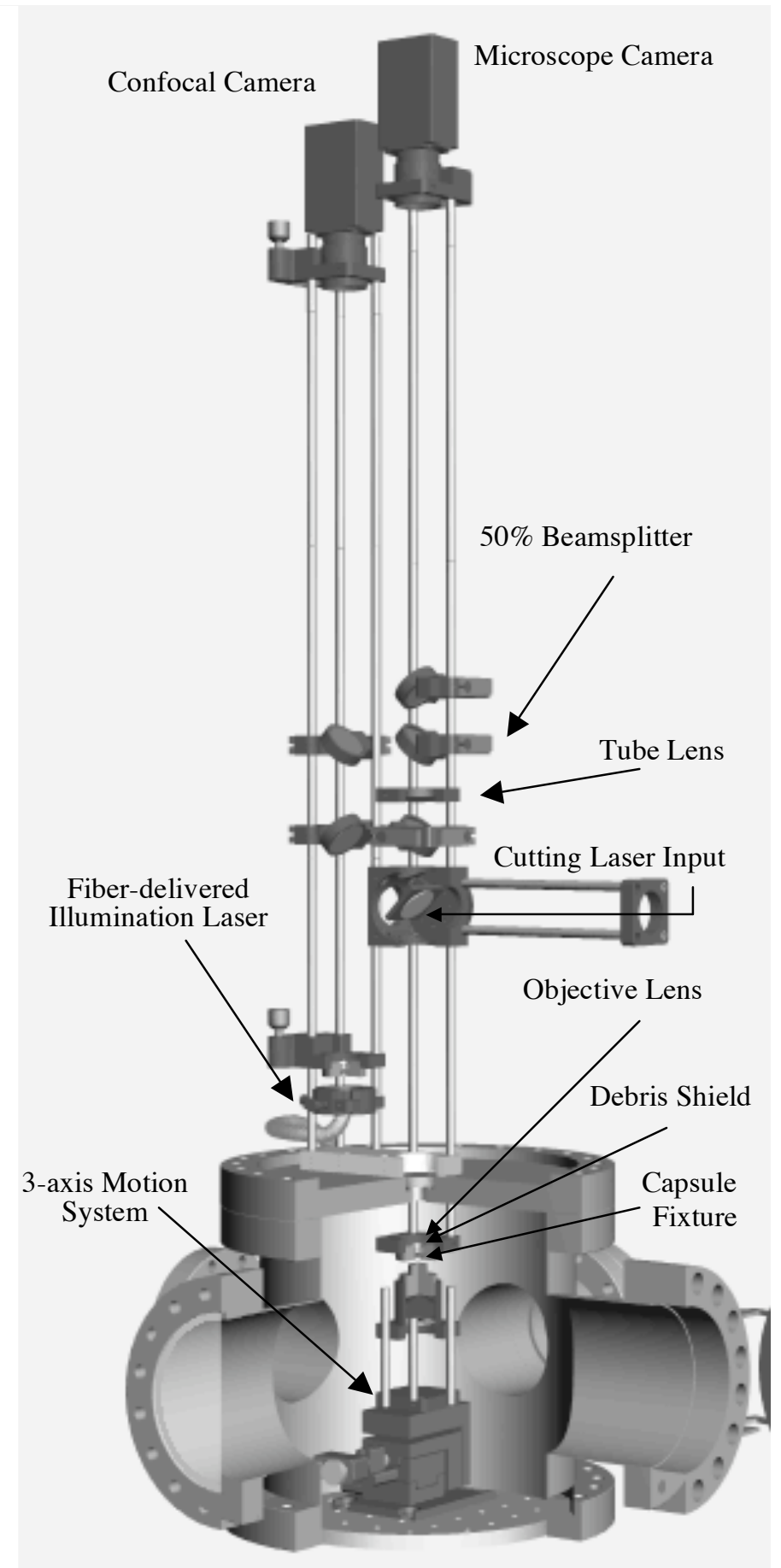

Figure 4. Micron-scale high aspect ratio hole drilling apparatus.

image. The appearance of the hole is a crucial indicator because the laser will drill only when the part is properly positioned, due to short range over which the laser intensity is above ablation threshold at such low pulse energies. The per-pulse energy is immediately ramped by rotating the waveplate at a speed of $1^{\circ}$ per second for 45 seconds to end at the maximum energy $(3.5 \mu \mathrm{J})$. The ramped energy-versus-time profile avoids placing large peak intensities on the part until a channel has formed that allows the increased energy required for breakthrough to be spread over the large surface area of the interior hole wall. Observing the microscope image during the ramp shows the hole enlarging with increasing energy. At the conclusion of the ramp the pulse energy stays constant while the laser is allowed to dwell on the part for an additional 2 minutes. At no time during the drilling does the part move spatially.

\section{RESULTS AND CHARACTERIZATION}

Well over 400 holes have been drilled in beryllium to date with more than 65 of those in capsules. Figure 5 shows typical entrance and exit holes obtained during a one-day repeatability campaign in $125 \mu \mathrm{m}$ thick Be foils. The hole-to-hole morphological repeatability in these foils was quite good, particularly for the entrance side. The 3 $\mu \mathrm{m}$ entrance through $125 \mu \mathrm{m}$ of material represented an aspect ratio of about 41 , which is significantly better than the 35 required by the application. Consistent with the waveguiding model discussed previously, the extra depth encountered with $175 \mu \mathrm{m}$ (full thickness) parts posed a greater challenge to the system due to the significant increase in attenuation within the longer waveguide. The extra loss was overcome by increasing the laser energy, which in turn enlarged the hole. Initial results in $175 \mu \mathrm{m}$ material demonstrated this effect, as shown in Figure 6.

The full depth drilling results showed an enlargement of the entrance hole to between five and seven microns. The exit morphology was also markedly different, although it is important to note that several months (and changes to the laser) separate the $125 \mu \mathrm{m}$ and the $175 \mu \mathrm{m}$ results. The bifurcation of the exit hole in to two separate channels was a surprising result, particularly given the high degree of repeatability. Near-field mode quality observations of the beam showed a two-lobe profile. A second sample was drilled with slightly different alignment of the laser relative to the final lens. These results showed a lesser degree of bifurcation. A third sample was drilled after adjustments to the laser removed the two-lobed profile. These results showed a further decrease in the degree of bifurcation and, in some instances, an elimination of it. Figure 7 shows samples of the exit holes obtained from each of the three experiments. Although bifurcation still existed in some of the most recent holes, there was no evidence of any features in a far field focal scan that would directly drive the bifurcation. 

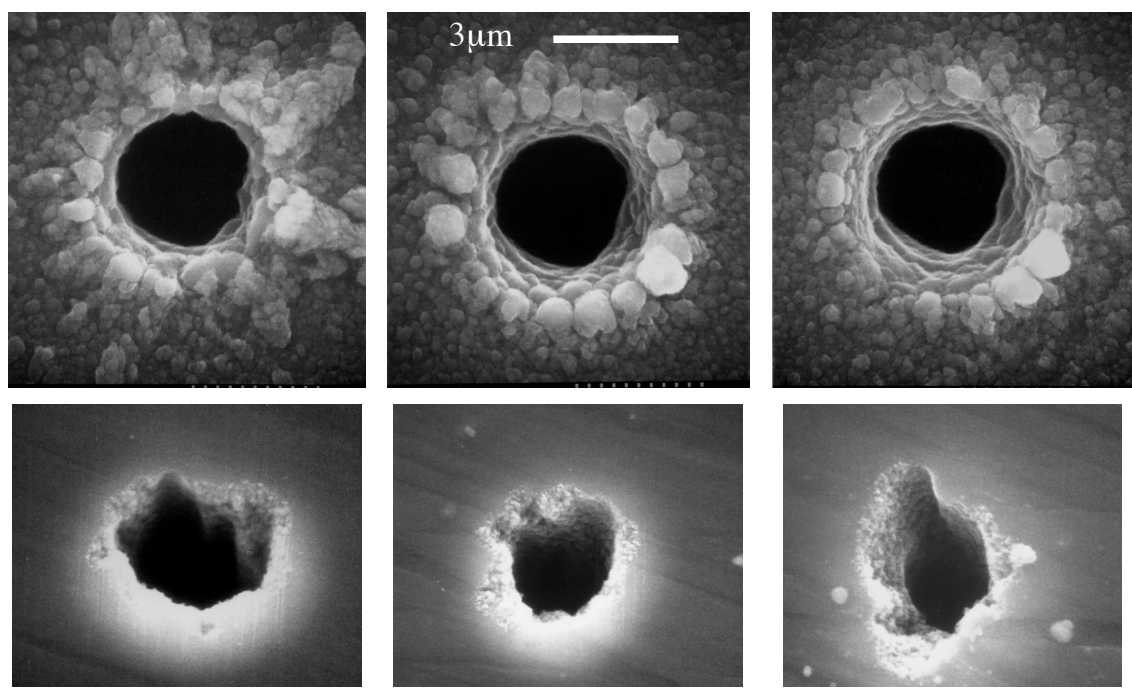

Figure 5. SEM micrographs of holes drilled in $125 \mu \mathrm{m}$ thick beryllium. Entrance holes are shown on top with their corresponding exits below. Scale bar is applicable to all pictures.
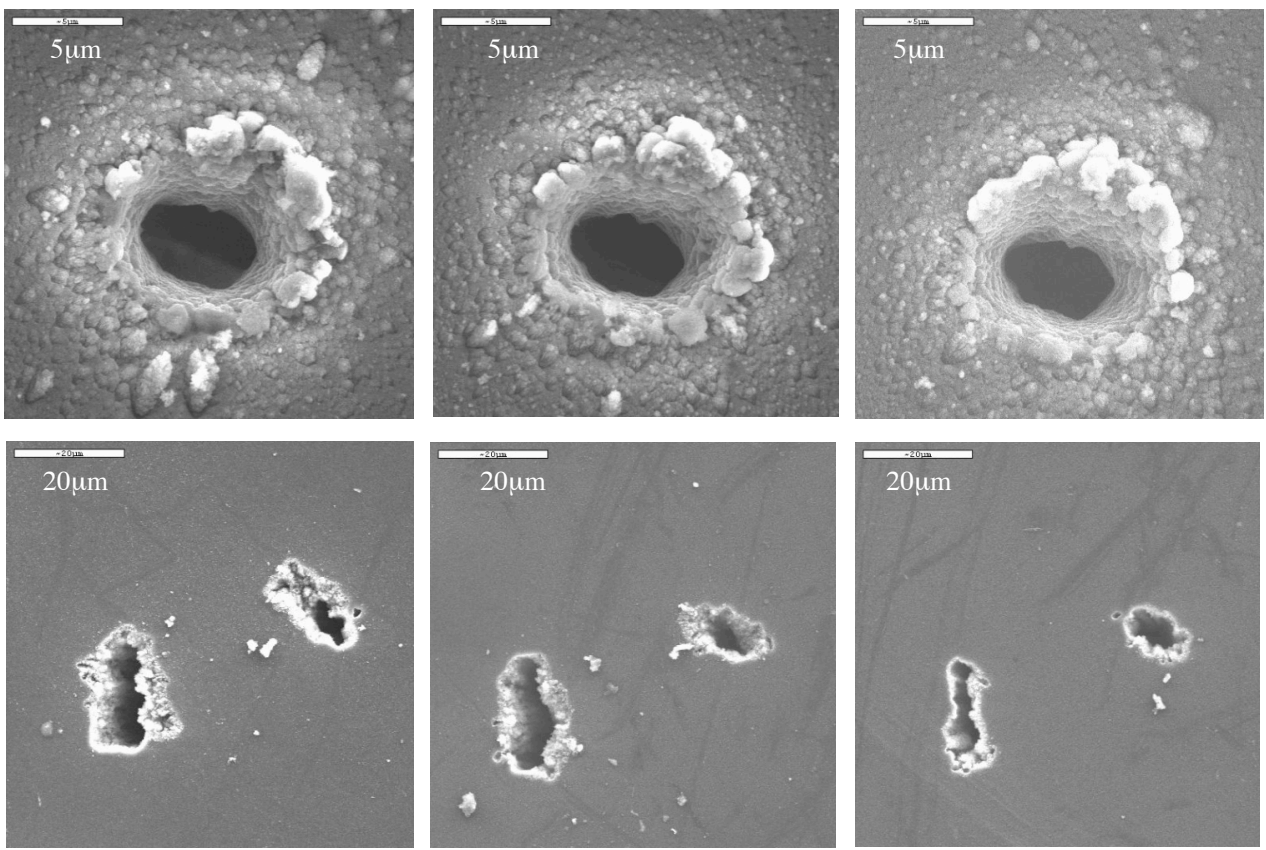

Figure 6: Representative entrance (top) and exit (bottom) holes produced in $175 \mu \mathrm{m}$ thick Be foil.
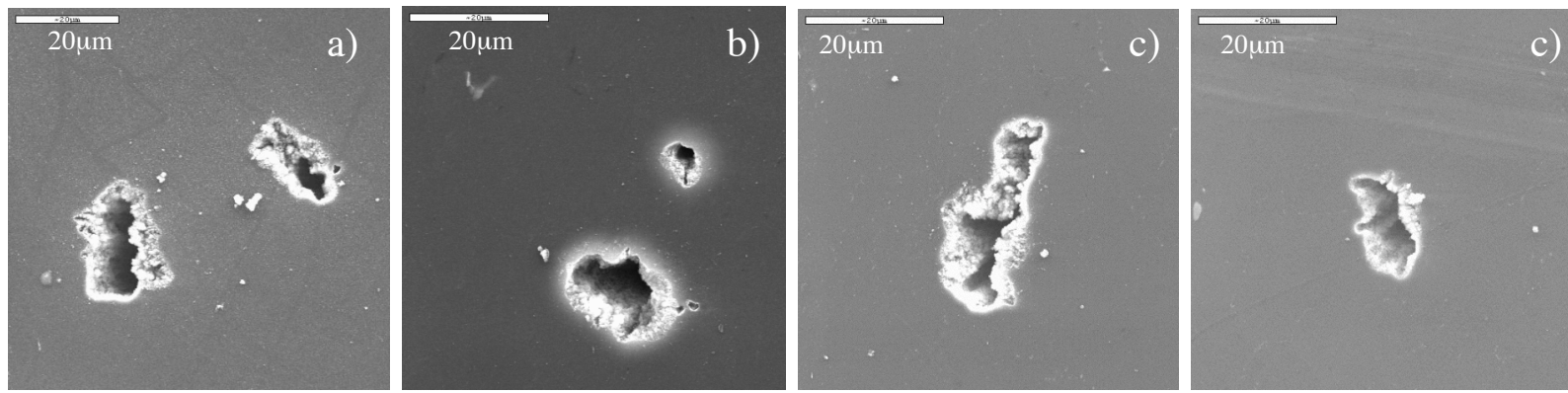

Figure 7. Exit hole evolution over multiple $175 \mu \mathrm{m}$ thick Be experiments. Exits shown are representative examples taken from sample 1 (a), sample 2 (b) and sample 3 (c). 
The collection of the full thickness results seems to suggest that the exit morphology is sensitive to the input laser mode quality and its alignment within the optical system. The need for a method to characterize the internal structure of the holes became apparent with these results since understanding how a hole transitions from a single entrance to the bifurcated exits is important. Understanding the internal morphology will also be important when attempting to determine the effects that varying process parameters has on the hole structure.

To be useful, the characterization of the internal hole structure must not alter that structure or introduce any additional features that did not already exist. Even if clean cross sections could be obtained without clogging, a simple two-dimensional planar view would not adequately characterize the complex three-dimensional structure that is suggested by the exit holes. The Chemistry and Materials Science (C\&MS) directorate recently obtained an instrument that provides the capability of high-resolution $\mathrm{x}$-ray radiography. This instrument is also capable of acquiring tomographic data to allow the complete digital reconstruction and subsequent measurement of the hole morphology at resolutions on the order of $100 \mathrm{~nm}$.

The instrument generates the diagnostic x-rays by bombarding an electron beam on a platinum target. These $\mathrm{x}$-rays are then directed toward a 1340x1300 element
CCD array. The object being radiographed is placed in the flux and the CCD acquires an image for a length of time suitable for the material and desired density contrast. This application used an acceleration voltage of $20 \mathrm{kV}$ during a 60 second exposure and then averaged five of those frames to produce a single image shown here. Figure 8 shows several $x$-ray images at various angles of some laser-drilled holes from the first full-thickness Be sample. This sample was chosen because the hole structure (bifurcation) provided interesting morphological features to demonstrate the capabilities of this diagnostic.

The x-ray images show many interesting internal features of the bifurcated holes. First, each hole exhibits a reduced diameter (2 $\mu \mathrm{m})$ "neck" that appears approximately $10 \mu \mathrm{m}$ below the surface. Knowing the depth of features such as these is important because any artifacts that are within $20 \mu \mathrm{m}$ of the surface are likely to be removed during the counterboring process for the fill tube. Although this neck seems to indicate that the beam was focused $10 \mu \mathrm{m}$ below the surface of the part, the fact that the entrance holes are properly sized confirm the part was positioned correctly. Other interesting features include the onset of bifurcation and the multiple exits that are clearly visible. The data are all the more revealing when the images, animations and reconstructions can be examined at the high resolution and contrast ratio of a computer monitor.
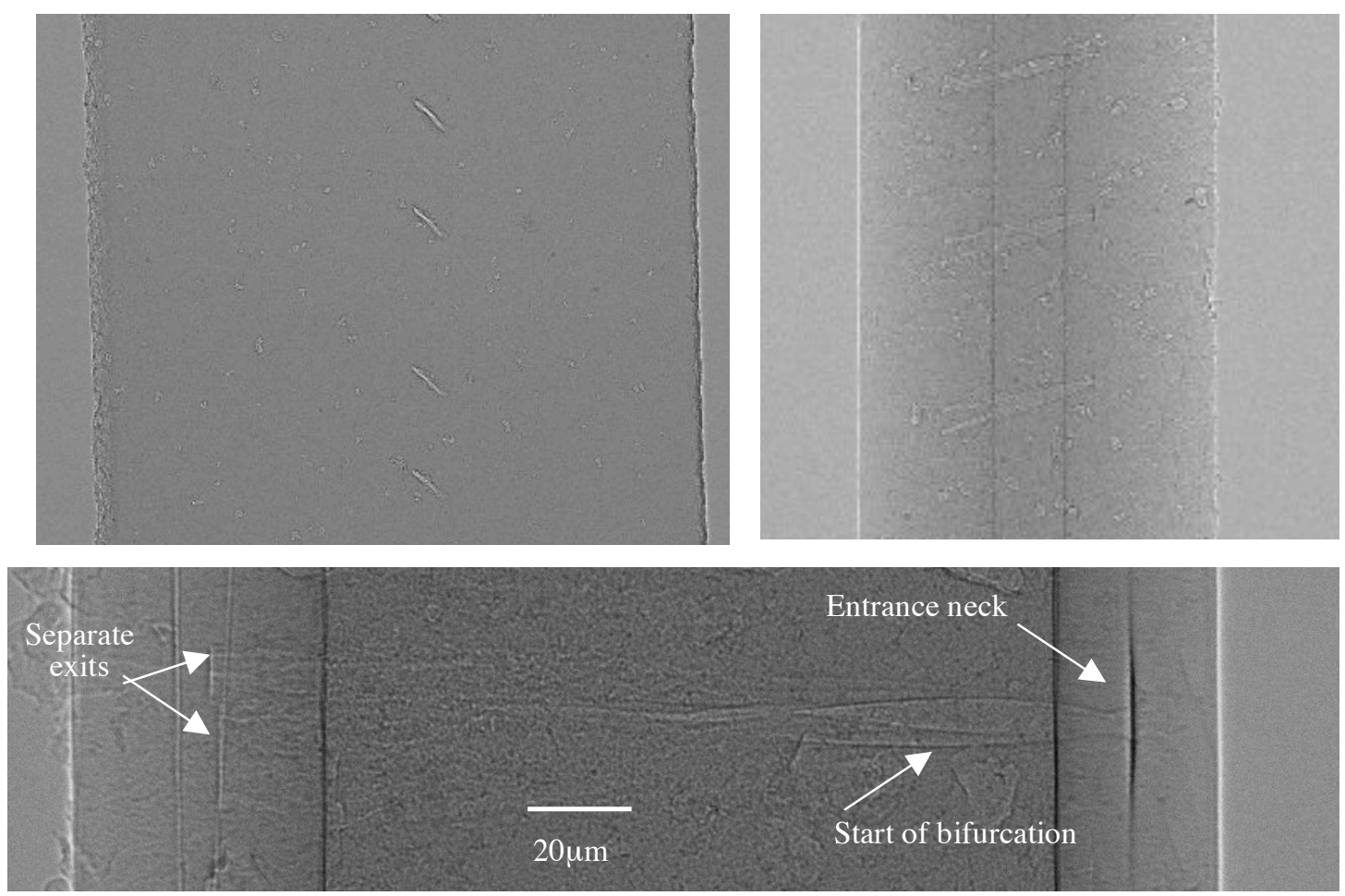

Figure 8. Typical x-ray microscopy images of $175 \mu \mathrm{m}$ thick planar beryllium foils showing the various attributes of the laser-drilled channels. 


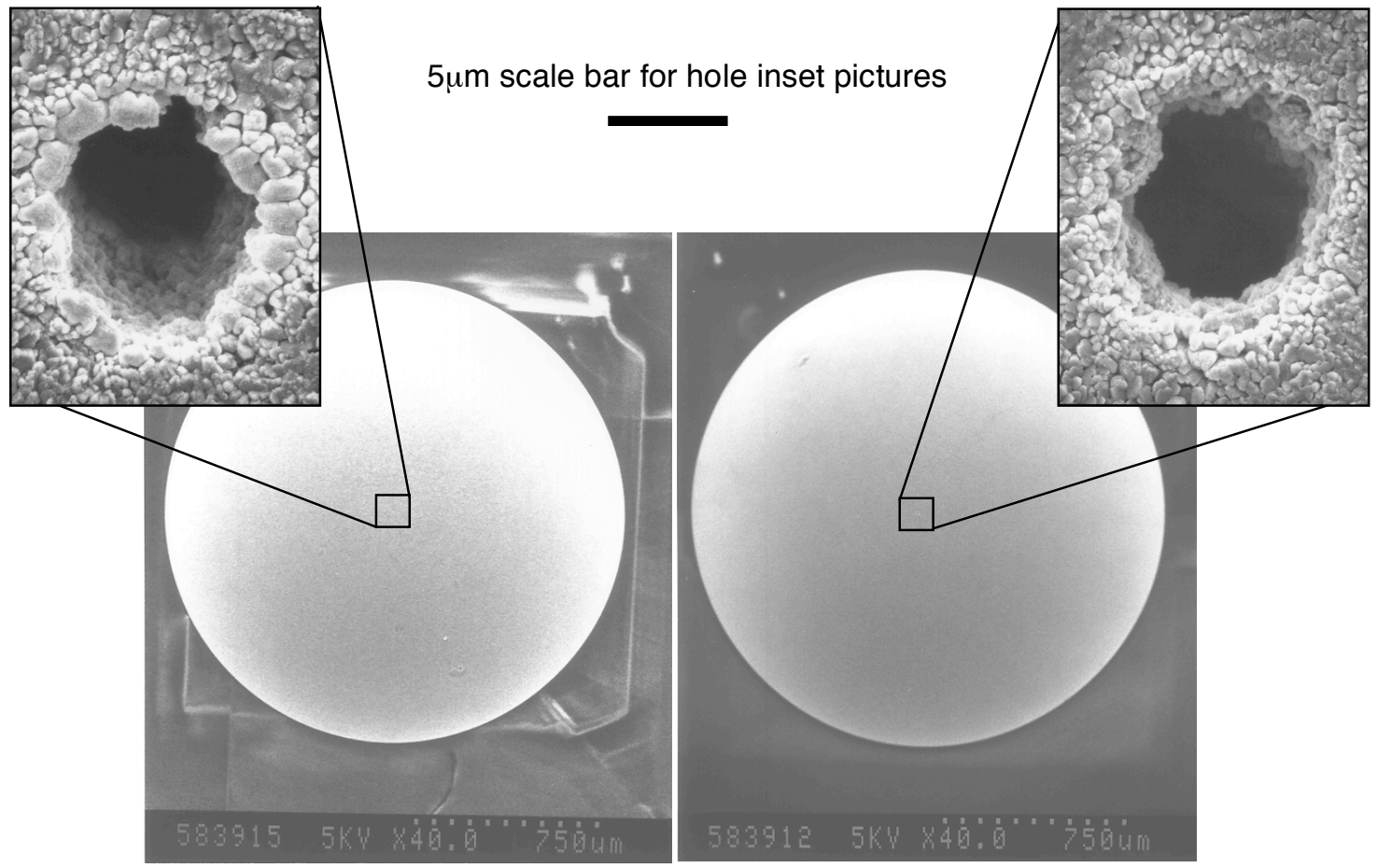

Figure 9. Representative holes drilled in Be capsules.

Much of the process development was done on planar Be samples for ease of fixturing and characterization. In addition, more than 65 capsules of various wall thicknesses (up to $175 \mu \mathrm{m}$ thick) have been drilled. Figure 9 shows representative samples of these results.

Spherical capsules present a challenge in characterization because one can not directly view the exit hole on the interior wall. In addition, $x$-ray microscopy presents difficulties in image contrast because the thickness of the material the $\mathrm{x}$-rays must traverse varies with the depth of the hole. This potentially leaves the entrance portion of the image overexposed while the exit area remains underexposed. Inferring the morphology of the capsule holes from planar results may not be valid since there are significant differences in the material grain size and grain alignment between that of the sputtered capsules and the rolled Be planar samples.

There are two general concerns when drilling holes in the Be capsules envisioned for ignitions shots. First, the hole must go through the full thickness of beryllium in addition to the $\mathrm{CH}$ mandrel located on the interior wall of the capsule. Secondly, the hole must have a sufficiently high conductance to allow for reasonable fill times.

Penetrating the $15 \mu \mathrm{m} \mathrm{CH}$ mandrel is important to allow for its subsequent pyrolization and removal. Initially we were concerned that the dielectric nature of $\mathrm{CH}$ would not provide the conditions necessary to waveguide and confine the light as it does in the metallic capsule. Therefore, it was possible that the light emerging from the exit of the Be capsule would rapidly diverge and drop below ablation threshold prior to drilling through the mandrel.

A series of experiments were conducted to determine if penetration of the $\mathrm{CH}$ was possible and how much extra time was required once breakthrough on the beryllium was complete. The experiments were performed on a 175 $\mu \mathrm{m}$ thick Be planar foil that was coated with the proper thickness of $\mathrm{CH}$. The sample was drilled using the standard drill tool, with the exception that the laser was shuttered after various time intervals once breakthrough of the beryllium was detected. Breakthrough detection was accomplished by placing a photodetector at the focal point of a lens that collected any light emerging from the back of the sample. The detector would produce a measurable voltage once we broke through the beryllium (as opposed to the $\mathrm{CH}$ material) because $\mathrm{CH}$ is adequately transparent to both the drilling and illumination wavelengths. Figure 10 shows a selection of the exit holes observed on the $\mathrm{CH}$ backer, demonstrating successful drilling of the $\mathrm{CH}$ material.

The inability to easily field a breakthrough diagnostic for capsules drove the need for a device to ascertain if the hole went all the way through the wall. Additionally, it would be useful to know if the fill channel was large enough to fuel through prior to allowing a capsule to 

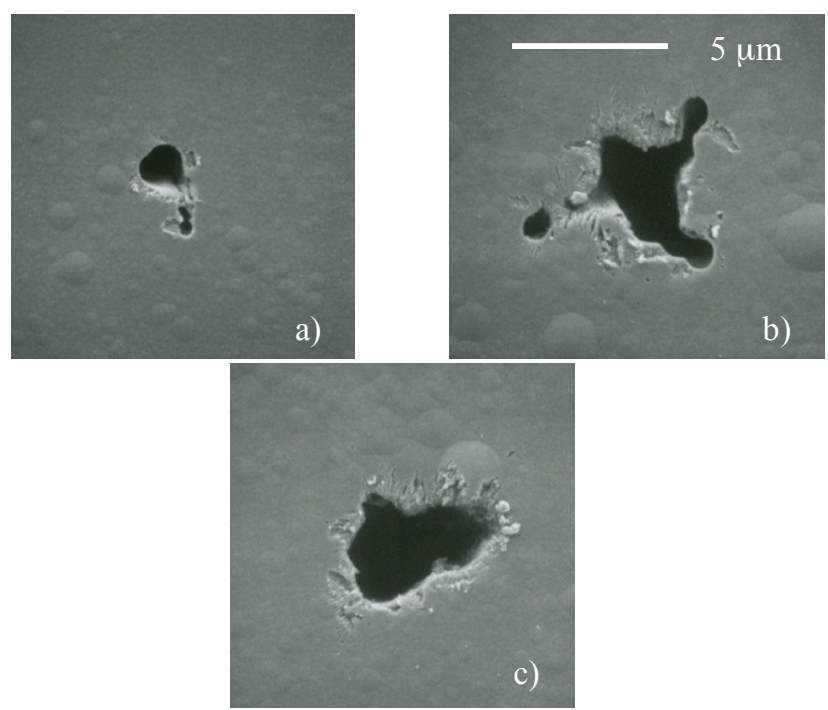

Figure 10. Exit holes in the $\mathrm{CH}$ backer after dwelling at full energy for $5 \sec (a), 30 \sec (b)$ and $120 \sec (c)$ after breakthrough of the Be foil. Scale applies to all pictures.

continue in the fabrication process. To this end a conductance measurement device was constructed to assist in determining the relative flow capacity of a given laser-drilled channel. ${ }^{20}$ This device monitored the timeresolved pressure inside a cavity that was barely larger than the capsule under test. A mechanically-actuated ram rapidly pressurized this cavity with air while a laser ranging gauge measured the displacement of a flexible membrane attached to one wall of the cavity. The deformation of the membrane is a direct measurement of the pressure within the apparatus. One would expect that a capsule with no conductance (un-drilled) would show a
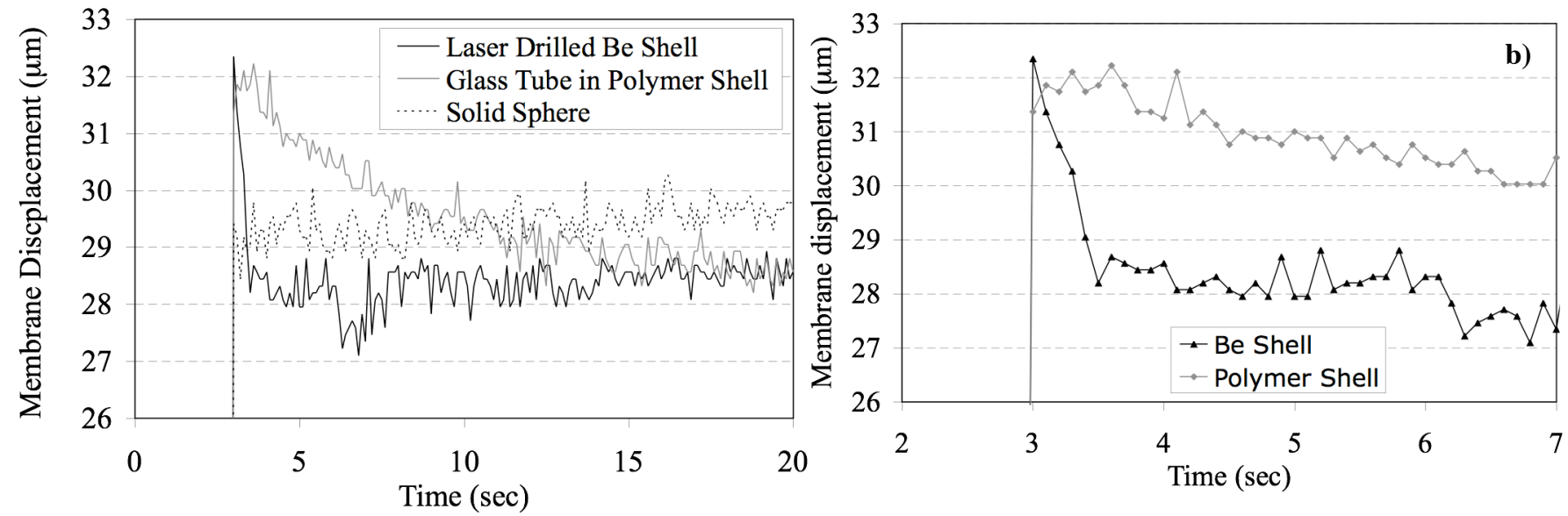

Figure 11. Time-resolved pressure profiles for various test capsules. Glass tube has an approximate inner diameter of $3 \mu \mathrm{m}$. Laser-drilled shell's entrance hole diameter was measured at $5 \mu \mathrm{m}$. The solid sphere had a slightly smaller diameter than either shell. The polymer shell and the Be shell both showed an initial displacement of about $32 \mu \mathrm{m}$, but with differing decay rates corresponding to the conductance. In contrast, the solid sphere remained near its initial displacement of $29.5 \mu \mathrm{m}$. The faster decay rate of the laser-drilled shell indicates that the fill hole had a larger conductance than the $3 \mu \mathrm{m}$ glass tube in the polymer shell. The expanded scale plot of the same data (b) shows that there is sufficient temporal resolution to acquire the rapidly decaying profile seen in the laser drilled Be shell. pressure increase to an initial value and remain there indefinitely. On the other hand, a capsule of the same diameter with a hole would pressurize to the same initial value and then decay to some lesser value as the internal volume of the capsule filled. Measurement of this decay time provides a means of determining the conductance of the fill hole.

Early tests of the conductance apparatus showed that it behaved as one would expect. The apparatus is currently undergoing characterization to match decay times with holes of known diameter. Figure 11 shows some initial tests, including a laser-drilled full thickness capsule. Although absolute conductance numbers can not yet be assigned to the profiles shown, the data indicate that the laser-drilled hole does allow gas transport on the time scales one would expect for $5 \mu \mathrm{m}$ holes.

\section{CONCLUSION}

A system was developed that enabled a 5-7 $\mu \mathrm{m}$ diameter fill hole to be drilled in the $175 \mu \mathrm{m}$ thick copperdoped beryllium ablator and $15 \mu \mathrm{m}$ thick $\mathrm{CH}$ mandrel of proposed ignition capsules for NIF. The system utilized femtosecond materials processing technology and relied on the optical confinement of the channel itself to drill well beyond the Rayleigh range of the focusing system. The exit morphology of the holes appeared to be sensitive produced complex internal hole shapes. We have demonstrated that it is possible to non-destructively characterize these holes by $\mathrm{x}$-ray microscopy or to the input laser characteristics that, in some instances, 
conductance measurements. Future experiments centering on laser mode quality, focusing conditions, focusing lens NA, material dependencies and pulse repetition frequency are under consideration.

\section{ACKNOWLEDGMENTS}

The authors thank Richard Shuttlesworth for mechanical design and fabrication, Richard Seugling for the conductance apparatus, Nick Teslich for providing $\mathrm{x}$ ray microscopy, Ed Lindsey for SEM analysis and Susan Haynes for process development drilling. Additional thanks to Andrew Forsman of General Atomics for feedback and laser characterization assistance. This work was performed under the auspices of the U.S. Department of Energy by University of California, Lawrence Livermore National Laboratory under Contract W-7405Eng-48.

\section{REFERENCES}

1. S.W. HAAN, et al., "Update on Specification for NIF Ignition Targets, and Their Rollup into an Error Budget," Fusion Sci. Technol. this issue.

2. G.H. MILLER, E.I. MOSES and C.R. WUEST, "The National Ignition Facility," Opt. Eng. 42, 2841 (2004).

3. J. EDWARDS, M. MARINAK, T. DITTRICH, S. HAAN, J. SANCHEZ, J. KLINGMANN and J. MOODY, "The Effects of Fill Tubes on the Hydrodynamics of Ignition Targets and Prospects for Ignition," Phys. of Plasmas 12, 056318 (2005).

4. Z. YU and K.P. RAJURKAR, "High Aspect Ratio and Complex Shaped Blind Micro Holes by Micro EDM," Annals of CIRP, 51, 359 (2002).

5. K. WANG, A. CHELNOKOV, S ROWSON, J.M. LOURTIOZ, "Extremely High-aspect-ratio Patterns in Macroporous Substrate by Focused-ion-beam Etching: The Realization of Three-dimensional Lattices," Appl. Phys. A 76, 1013 (2003).

6. M. MCELFRESH, et al., "Fabrication of Beryllium Capsules with Copper-doped Layers for NIF Target: A Progress Report,” Fusion Sci. Technol. this issue.

7. H. XU, et al., "Be Coatings on Spherical Surface for NIF Target Development," Fusion Sci. Technol. this issue.

8. B.W. MCQUILLAN, et al., "The PaMS/GDP Process for Production of ICF Target Mandrels," Fusion Technol. 31, 381 (1997).

9. R.C. COOK, S.A. LETTS, S.R. BUCKLEY and E.M. FEARON, "Pyrolitic Removal of the Plastic Mandrel from Sputtered Beryllium Shells," Fusion Sci. Technol. this issue.
10. B.C. STUART, M.D. FEIT, S. HERMAN, A.M. RUBENCHIK, B.W. SHORE and M.D PERRY, "Optical Ablation by High-powered Short-pulse Lasers," J. Opt. Soc. Am. B 13, 459 (1996).

11. B.N. CHICHKOV, C. MOMMA, S. NOLTE, F. VON ALVENSLEBEN, and A. TUNNERMANN, "Femtosecond, Picosecond and Nanosecond Laser Ablation of Solids," Appl. Phys. A 63, 109 (1996).

12. S. NOLTE, C. MOMMA, H. JACOBS, A. TUNNERMANN, B.N. CHICHKOV, B. WELLEGEHAUSEN and H. WELLING, "Ablation of Metals by Ultrashort Laser Pulses," J. Opt. Soc. Am. B 14, 2716 (1997).

13. M.D. PERRY, B.C. STUART, P.S. BANKS, M.D. FEIT, V. YANOVSKY and A.M. RUBENCHIK, "Ultrashort-pulse Laser Machining of Dielectric Materials," J. Applied Physics 85, 6803 (1999).

14. X. ZHU, A.YU. NAUMOV, D.M. VILLENEUVE, and P.B. CORKUM, "Influence of Laser Parameters and Material Properties on Micro Drilling with Femtosecond Laser Pulses," Appl. Phys A 69 [suppl.], S367 (1999).

15. A.E. WYNNE and B.C. STUART, "Rate Dependence of Short-pulse Laser Ablation of Metals in Air and Vacuum," Applied Physics A 76, 373 (2003).

16. P.S. BANKS, M.D. FEIT, A.M. RUBENCHIK, B.C. STUART and M.D. PERRY, "Material Effects in Ultra-short Pulse Laser Drilling of Metals," Appl. Phys. A 69 [suppl.], S377 (1999).

17. V.N. ANISIMOV, L.A. BOL'SHOV, K.A. KRIVORUCHKO, D.D. MALYUTA, V.P. RESHETIN, A YU. SEBRANT and R.I. SOLOUKHIN, "Absorption of Infrared Radiation in Metallic Capillaries," Sov. J. Quantum Electron. 17 101, (1987).

18. D. STRICKLAND and G. MOUROU, "Compression of Amplified Chirped Optical Pulses," Opt. Comm 56, 219 (1985).

19. S. NOLTE, C. MOMMA, G. KAMLAGE, A. OSTENDORF, C. FALLNICH, F. VON ALVENSLEBEN, and H. WELLING, "Polarization Effects in Ultrashort-pulse Laser Drilling," Appl. Phys. A 68, 563 (1999).

20. R.M. SEUGLING, W.W. NEDERBRAGT, J.L. KLINGMANN, S. EDSON and R. COOK, "Cryogenic Capsule Fill Hole Conductance Measurement," Sixteenth Target Fabrication Specialists Meeting, Poster t-p34 (2005). 